\title{
ON THE DISPLACEMENT OF EQUILIBRIUM
}

\section{BY PAUL SAUREL}

It is well known that among the consequences of the phase rule are the following theorems: The state of equilibrium of an invariant system is completely determined if its entropy and its volume be given; the state of equilibrium of a univariant system is completely determined if any one of the three pairs of variables: entropy and volume, temperature and volume, entropy and pressure be given ; and, fnally, the state of equilibrium of a bivariant or multivariant system is completely determined if any one of the four pairs of variables: entropy and volume, temperature and volume, entropy and pressure, temperature and pressure be given. The object of the present note is to show that to each of the above pairs of variables there correspond two theorems relating to the displacement of equilibrium.

As is well known, the two fundamental principles of thermodynamics may be expressed by the following relations :

$$
\begin{gathered}
d \mathrm{E}=d \mathrm{Q}-\mathrm{\Pi} d \mathrm{~V}, \\
d \mathrm{Q} \leqq \mathrm{T} d \mathrm{H},
\end{gathered}
$$

in which $\mathrm{E}, \mathrm{H}, \mathrm{V}, \mathrm{T}, \Pi$ denote respectively the energy, entropy, volume, temperature, and pressure of the system, and $d Q$ denotes the heat absorbed during a small change. In the second of the above relations the sign of equality applies to reversible changes while the sign of inequality applies to real changes. As a consequence of the above relations we obtain at once

$$
d \mathrm{E} \leqq \mathrm{T} d \mathrm{H}-\Pi d \mathrm{~V} .
$$

If we define the three functions: $F$, the free energy, $G$, the heat function, and $\Phi$, the thermodynamic potential, by means of the equations

$$
\begin{aligned}
& \mathrm{F}=\mathrm{E}-\mathrm{TH}, \\
& \mathrm{G}=\mathrm{E}+\Pi \mathrm{V}, \\
& \Phi=\mathrm{E}-\mathrm{TH}+\Pi \mathrm{V},
\end{aligned}
$$

we obtain from 3 without difficulty 


$$
\begin{aligned}
& d \mathrm{~F} \leqq-\mathrm{H} d \mathrm{~T}-\Pi d \mathrm{~V}, \\
& d \mathrm{G} \leqq \mathrm{T} d \mathrm{H}+\mathrm{V} d \Pi, \\
& d \Phi \leqq-\mathrm{H} d \mathrm{~T}+\mathrm{V} d \Pi .
\end{aligned}
$$

From 3, 4, 5, 6 it follows that the condition of stable equilibrium can be expressed in any one of the four forms :

$$
\begin{aligned}
& (\Delta \mathrm{E})_{\mathrm{H}, \mathrm{v}}>0, \\
& (\Delta \mathrm{F})_{\mathrm{r}, \mathrm{v}}>0, \\
& (\Delta \mathrm{G})_{\mathrm{H}, \Pi}>0, \\
& (\Delta \Phi)_{\mathrm{T}, \Pi}>0 .
\end{aligned}
$$

In each of these inequalities the quantity in parenthesis denotes the variation due to a virtual change during which the mass of the system and the variables written as subscripts are kept constant.

Let us denote by $\mathrm{M}_{i j}$ the mass of the $j$-th component which is present in the $i$-th phase, and let us denote by $r$ the number of phases and by $n$ the number of independent components. Then inequality 7 can be written in the form

$$
\sum_{i=1}^{r} \sum_{j=1}^{n} \frac{\partial \mathrm{E}_{1}}{\partial \mathrm{M}_{i j}} \delta \mathrm{M}_{i j}+\frac{1}{2} \sum_{i=1}^{r} \sum_{j=1}^{n} \sum_{k=\mathrm{I}}^{n} \frac{\partial^{2} \mathrm{E}}{\partial \mathrm{M}_{i j} \partial \mathrm{M}_{i k}} \delta \mathrm{M}_{i j} \delta \mathrm{M}_{i k}>0 .
$$

If all the virtual changes are reversible we may replace each variation $\delta \mathrm{M}_{i j}$ by its negative; we thus obtain

$$
-\sum_{i=\mathrm{I}}^{r} \sum_{j=\mathrm{I}}^{n} \frac{\partial \mathrm{E}_{1}}{\partial \mathrm{M}_{i j}} \delta \mathrm{M}_{i j}+\frac{1}{2} \sum_{i=\mathrm{I}}^{r} \sum_{j=\mathrm{I}}^{n} \sum_{k=\mathrm{I}}^{n} \frac{\partial^{2} \mathrm{E}}{\partial \mathrm{M}_{i j} \partial \mathrm{M}_{i k}} \delta \mathrm{M}_{i j} \delta \mathrm{M}_{i k}>0 .
$$

From II and 12 it follows without difficulty that

$$
\sum_{i=1}^{r} \sum_{j=1}^{n} \frac{\partial \mathrm{E}}{\partial \mathrm{M}_{i j}} \delta \mathrm{M}_{i j}=0, \quad \sum_{i=1}^{r} \sum_{j=1}^{n} \sum_{k=\mathrm{I}}^{n} \frac{\partial^{2} \mathrm{E}}{\partial \mathrm{M}_{i j} \partial \mathrm{M}_{i k}} \delta \mathrm{M}_{i j} \delta \mathrm{M}_{i k}>0 .
$$

In like manner we can replace 8,9 and Io by the following pairs of conditions : 


$$
\begin{aligned}
& \sum_{i=1}^{r} \sum_{j=1}^{n} \frac{\partial \mathrm{F}}{\partial \mathrm{M}_{i j}} \delta \mathrm{M}_{i j}=0 \\
& \sum_{i=1}^{r} \sum_{j=1}^{n} \sum_{k=1}^{n} \frac{\partial^{2} \mathrm{~F}}{\partial \mathrm{M}_{i j} \partial \mathrm{M}_{i k}} \delta \mathrm{M}_{i j} \delta \mathrm{M}_{i k}>0 ; \\
& \sum_{i=1}^{r} \sum_{j=\mathrm{I}}^{n} \frac{\partial \mathrm{G}}{\partial \mathrm{M}_{i j}} \delta \mathrm{M}_{i j}=0, \\
& \sum_{i=1}^{r} \sum_{j=1}^{n} \sum_{k=1}^{n} \frac{\partial^{2} \mathrm{G}}{\partial \mathrm{M}_{i j} \partial \mathrm{M}_{i k}} \delta \mathrm{M}_{i j} \delta \mathrm{M}_{i k}>0 \\
& \sum_{i=\mathrm{I}}^{r} \sum_{j=\mathrm{I}}^{n} \frac{\partial \Phi}{\partial \mathrm{M}_{i j}} \delta \mathrm{M}_{i j}=0 \\
& \sum_{i=1}^{r} \sum_{j=1}^{n} \sum_{k=1}^{n} \frac{\partial^{2} \Phi}{\partial \mathrm{M}_{i j} \partial \mathrm{M}_{i k}} \delta \mathrm{M}_{i j} \delta \mathrm{M}_{i k}>0 .
\end{aligned}
$$

The first relation in each of the four pairs of relations I 3 , I4, I 5 and I 6 may be called the condition of equilibrium; the condition of equilibrium serves to establish the various theorems included in the phase rule. The second relation in each of the above pairs of relations may be called the condition of stability. Corresponding to each form of the condition of stability there are two theorems relating to the displacement of equilibrium. These theorems we shall now deduce.

Consider a system which is in stable equilibrium under a volume $\mathrm{V}$ and with an entropy $\mathrm{H}$. Conditions $\mathrm{I} 3$ are satisfied. If we increase the entropy and the volume by $d \mathrm{H}$ and $d \mathrm{~V}$, a new state of equilibrium establishes itself. In passing from the first state of equilibrium to the second the mass $M_{i j}$ receives an increment $d \mathrm{M}_{i j}$. The first of conditions I 3 must be satisfied when we replace $\mathrm{H}, \mathrm{V}, \mathrm{M}_{i j}$ by $\mathrm{H}+d \mathrm{H}, \mathrm{V}+d \mathrm{~V}, \mathrm{M}_{i j}+d \mathrm{M}_{i j}$. Making these substitutions we obtain without difficulty

$$
\begin{aligned}
d \dot{\mathrm{H}} \sum_{i=\mathrm{I}}^{r} \sum_{j=\mathrm{I}}^{n} \frac{\partial^{2} \mathrm{E}}{\partial \mathrm{H} \mathrm{M}_{i j}} \delta \mathrm{M}_{i j} & +d \mathrm{~V} \sum_{i=\mathrm{I}}^{r} \sum_{j=\mathrm{I}}^{n} \frac{\partial^{2} \mathrm{E}}{\partial \mathrm{V} \partial \mathrm{M}_{i j}} \delta \mathrm{M}_{i j} \\
+ & \sum_{i=\mathrm{I}}^{r} \sum_{j=\mathrm{I}}^{n} \sum_{k=\mathrm{I}}^{n} \frac{\partial^{2} \mathrm{E}}{\partial \mathrm{M}_{i j} \partial \mathrm{M}_{i k}} \delta \mathrm{M}_{i j} d \mathrm{M}_{i k}=0 .
\end{aligned}
$$

If we refer to equation 3 we find that when the system is in equilibrium

$$
\frac{\partial E}{\partial H}=T, \quad \frac{\partial E}{\partial V}=-\Pi .
$$

From these equations it follows that 


$$
\sum_{i=1}^{r} \sum_{j=1}^{n} \frac{\partial^{2} \mathrm{E}}{\partial \mathrm{H}_{\partial} \mathrm{M}_{i j}} \delta \mathrm{M}_{i j}=\delta \mathrm{T}, \quad \sum_{i=1}^{r} \sum_{j=1}^{n} \frac{\delta^{2} \mathrm{E}}{\partial \operatorname{VD}_{i j} \mathrm{M}_{i j}} \delta \mathrm{M}_{i j}=-\delta \Pi,
$$

in which $\delta \mathrm{T}$ and $\delta \Pi$ denote the changes which would have occurred in the temperature and the pressure, if, with constant entropy and under constant volume, the masses $M_{i j}$ alone had varied. If we suppose that $\delta \mathrm{M}_{i j}=d \mathrm{M}_{i j}$, in other words, if we suppose that the virtual changes in the masses are the same as the actual changes which occur when the system passes from one state of equilibrium to an adjoining state of equilibrium, it follows from the second of conditions I 3 that the third term in equation $I 7$ is positive. Equation $I 7$ can accordingly be written in the form

$$
\delta \mathrm{T} \cdot d \mathrm{H}-\delta \Pi \cdot d \mathrm{~V}<0 .
$$

Similar reasoning applied to conditions 14 , I 5 and 16 yields without difficulty the following inequalities:

$$
\begin{array}{r}
-\delta \mathrm{H} \cdot d \mathrm{~T}-\delta \Pi \cdot \delta \mathrm{V}<\mathrm{o}, \\
\delta \mathrm{T} \cdot d \mathrm{H}+\delta \mathrm{V} \cdot d \Pi<0, \\
-\delta \mathrm{H} \cdot d \mathrm{~T}+\delta \mathrm{V} \cdot d \Pi<\mathrm{o} .
\end{array}
$$

Inequality I. applies to all systems which are in stable equilibrium under a given volume and with a given entropy; it therefore applies to all systems whose variance is equal to or greater than zero. Inequality II. applies to all systems which are in stable equilibrium at a given temperature and under a given volume; it therefore applies to all systems whose variance is equal to or greater than one. Inequality III. applies to all systems which are in stable equilibrium under a given pressure and with a given entropy; it therefore applies to all systems whose variance is equal to or greater than one. Finally, inequality IV. applies to all systems which are in stable equilibrium at a given temperature and under a given pressure; it therefore applies to all systems whose variance is equal to or greater than two.

From each of the inequalities I., II., III. and IV. we can obtain without difficulty two theorems relating to the displace- 
ment of equilibrium. Thus from I. we get the two pairs of conditions :

$$
\begin{array}{lll}
d \mathrm{~V}=0, & \delta^{\prime} \mathrm{T} \cdot d \mathrm{H}<0 ; & \text { I. }(a) \\
d \mathrm{H}=0, & \delta \mathrm{II} \cdot d \mathrm{~V}>0 . & \text { I. }(b)
\end{array}
$$

In like manner from II., III. and IV. we get the pairs of conditions

$$
\begin{array}{lll}
d \mathrm{~V}=0, & \delta \mathrm{H} \cdot d \mathrm{~T}>0 ; & \text { II. }(a) \\
d \mathrm{~T}=0, & \delta \Pi \cdot d \mathrm{~V}>0 ; & \text { II. }(b) \\
d \Pi=0, & \delta \mathrm{T} \cdot d \mathrm{H}<0 ; & \text { III. }(a) \\
d \mathrm{H}=0, & \delta \mathrm{V} \cdot d \Pi<0 ; & \text { III. }(b) \\
d \Pi=0, & \delta \mathrm{H} \cdot d \mathrm{~T}>0 ; & \text { IV. }(a) \\
d \mathrm{~T}=0, & \delta \mathrm{V} \cdot d \Pi<0 . & \text { IV. }(b)
\end{array}
$$

These eight pairs of inequalities fall naturally into the four following groups :

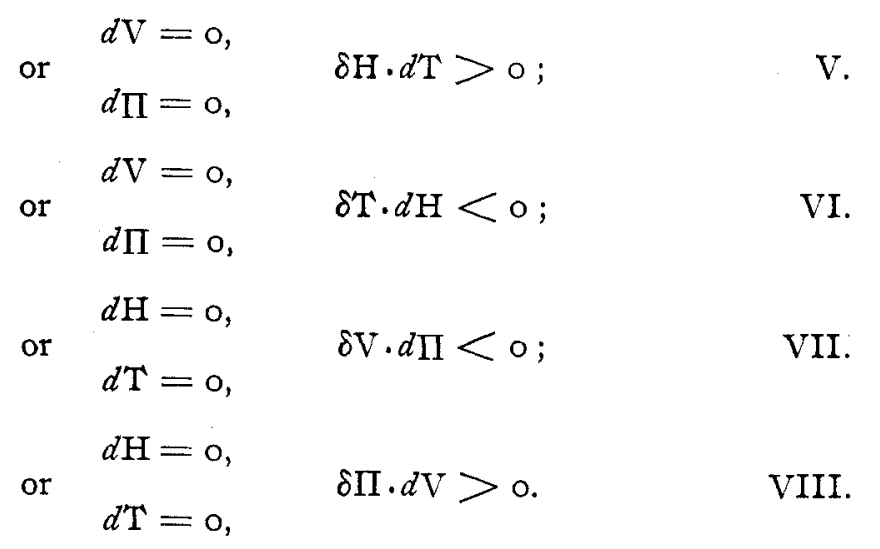

Conditions V. stated in words give us van't Hoff's theorem: If, under constant volume (or under constant pressure) the temperature of a system in equilibrium be increased, there results a change in the masses $M_{i j}$, or as we shall say, a change in the state of the system, which, if it were to take place under constant volume (or under constant pressure) and at constant temperature, would have for effect an increase of entropy. Similarly, if the temperature be lowered the resulting change in the state of the system would correspond to a decrease of entropy. 
Conditions VII. stated in words give us Le Chatelier's theorem : If, with constant entropy (or at constant temperature) the pressure of a system in equilibrium be increased, there results a change in the state of the system which, if it were to take place with constant entropy (or at constant temperature) and under constant pressure, would have for effect a decrease in volume. Similarly, if the pressure be decreased the resulting change in the state of the system would correspond to an increase in volume.

Conditions VI. give us the following theorem which is analogous to van't Hoff's theorem: If, under constant volume (or under constant pressure) the entropy of a system in equilibrium be increased, there results a change in the state of the system which, if it were to take place under constant volume (or under constant pressure) and with constant entropy, would have for effect a decrease in temperature. Similarly, if the entropy be decreased the resulting change in the state of the system would correspond to an increase in temperature.

Conditions VIII. give us the following theorem which is analogous to Le Chatelier's theorem: If, with constant entropy (or at constant temperature) the volume of a system in equilibrium be increased, there results a change in the state of the system which, if it were to take place with constant entropy (or at constant temperature) and under constant volume, would have for effect an increase in pressure. Similarly, if the volume be decreased the resulting change in the state of the system would correspond to a decrease in pressure.

It may not be out of place to recall briefly the principal dates in the history of the above theorems. In I 884 van't Hoff gave $^{x}$ the theorem which is expressed by conditions II.(a), and in the same year Le Chatelier gave, ${ }^{2}$ without demonstration, a theorem which was avowedly a generalization of van't Hoff's theorem and which in fact is equivalent to the theorems expressed by conditions IV.(a) and IV.(b). A few years later

1 Études de Dynamique, p. I6I (I884).

${ }^{2}$ Compt. rend. 99,786 (1884). 
Le Chatelier ${ }^{x}$ gave to the theorems enunciated by him a somewhat more definite form and asserted ${ }^{2}$ that the theorems concerning the displacement of equilibrium are consequences of the stability of the equilibrium of a system. In $\mathrm{r} 890$ Duhem made good this assertion by showing ${ }^{3}$ that the theorems corresponding to conditions II. (a), IV.(a) and IV.(b) are consequences of the following theorems of Gibbs: ${ }^{4}$ when a system is in stable equilibrium at a given temperature and under a given volume the free energy has a minimum value; when a system is in stable equilibrium at a given temperature and under a given pressure the thermodynamic potential has a minimum value. In $189 \mathrm{I}$ Le Chatelier and Mouret gave a demonstration ${ }^{5}$ of inequality IV. and deduced from it the two theorems IV. $(a)$ and IV.(b). In r 897 Duhem gave ${ }^{6}$ the theorem which corresponds to conditions II.(b). Finally, in 1897 , Planck gave ${ }^{7}$ an equation analogou's to equation $\mathrm{I} 7$ without, however, deducing the theorems IV. $(a)$ and IV.(b) which are consequences of it. The demonstration which we have just given of inequalities I., II., III. and IV. is identical with the demonstration which we have previously given ${ }^{8}$ of inequality IV.

Since the above was written there has appeared a note by Jouguet

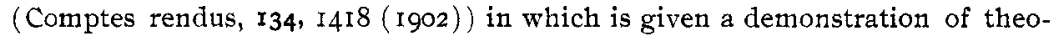
rems I. $(a), \mathrm{I} .(b)$, III. $(a)$ and $\operatorname{III}(b)$. This demonstration is in all essentials the saine as the one given above.

New York, June 14, rgoz.

${ }^{1}$ Recherches expérimentales et théoriques sur les Equilibres chimiques, pp. 48, 210 (1888).

${ }^{2}$ Recherches experimentales et theoriques sur les Equilibres chimiques, p. 58 (I 888 ).

${ }^{3}$ Annales de la Faculté de Toulouse, 4, N; (1890). Cf. Traité élémentaire de Mécavique chimique, I, I45, I84 (I897).

${ }^{4}$ On the Equilibrium of Heterogeneous Substances, pp. I45, r47.

${ }^{5}$ Les Equilibres chimiques, p. 29 (1891). Extrait de la Revue Générale des Sciences, 28 février et 15 mars, $189 \mathrm{I}$.

6 Traité élémentaire de Mécanique chimique, I, I53 (I897).

7 Thermodynamik, p. I76 (I897).

${ }^{8}$ Jour. Phys. Chem, 5, 6I (IgOI). 